\title{
Metformin and its therapeutic applications in autoimmune inflammatory rheumatic disease
}

\author{
Ji-Won Kim', Jung-Yoon Choe', and Sung-Hwan Park ${ }^{2}$
}

\begin{abstract}
${ }^{1}$ Division of Rheumatology, Department of Internal Medicine, Daegu Catholic University School of Medicine, Daegu; ${ }^{2}$ Division of Rheumatology, Department of Internal Medicine, Seoul St. Mary's Hospital, College of Medicine, The Catholic University of Korea, Seoul, Korea
\end{abstract}

Received : August 9, 2021

Accepted: September 23, 2021

Correspondence to

Sung-Hwan Park, M.D.

Division of Rheumatology,

Department of Internal Medicine,

Seoul St. Mary's Hospital, College

of Medicine, The Catholic

University of Korea, 222 Banpo-

daero, Seocho-gu, Seoul 06591,

Korea

Tel: +82-2-2258-6011

Fax: +82-2-599-3589

E-mail: rapark@catholic.ac.kr

https://orcid.org/0000-0003-1711-

2060
Metformin is a first-line therapeutic agent for type 2 diabetes. Apart from its glucose-lowering effect, metformin is attracting interest regarding possible therapeutic benefits in various other conditions. As metformin regulates cell metabolism, proliferation, growth, and autophagy, it may also modulate immune cell functions. Given that metformin acts on multiple intracellular signaling pathways, including adenosine monophosphate (AMP)-activated protein kinase (AMPK) activation, and that AMPK and its downstream intracellular signaling control the activation and differentiation of $T$ and $B$ cells and inflammatory responses, metformin may exert immunomodulatory and anti-inflammatory effects. The efficacy of metformin has been investigated in preclinical and clinical studies on rheumatoid arthritis, osteoarthritis, systemic lupus erythematosus, Sjögren's syndrome, scleroderma, ankylosing spondylitis, and gout. In this review, we discuss the potential mechanisms through which metformin exerts its therapeutic effects in these diseases, focusing particularly on rheumatoid arthritis and osteoarthritis.

Keywords: Metformin; Arthritis, rheumatoid; Osteoarthritis; Connective tissue diseases; AMP-activated protein kinases

\section{INTRODUCTION}

Metformin, a first-line therapeutic agent for the treatment of type 2 diabetes, is a biguanide synthetically derived from glucose-lowering herbal medicines. Although its efficacy and safety in type 2 diabetes have been known for decades, the molecular mechanisms of metformin remain under investigation. Studies have found that multiple modes of action are involved in the glucose-lowering effect of metformin [1]. Metformin acts on adenosine monophosphate (AMP)-activated protein kinase (AMPK)-dependent and -independent mechanisms to reduce hepatic glucose production and increase peripheral glucose utilization. AMPK activation of hepatocytes is induced by metformin via reduced cellular energy or upstream AMPK kinase, leading to downregulation of gluconeogenic gene transcription $[2,3]$. Metformin targets mitochondrial respiratory chain complex 1 to decrease adenosine triphosphate (ATP) and increase the AMP/ATP ratio, and is thereby linked to AMPK activation [4-6]. AMPK-independent mechanisms are also thought to play a role in the regulatory action of metformin on hepatic gluconeogenesis, such as the inhibition of gluconeogenic enzymes, suppression of glucagon signaling, and alteration of a cellular redox state by direct inhibition of the mitochondrial enzymes involved in electron transport shuttle [7-10].

AMPK is a regulator of cellular metabolism that senses 
cellular energy status. AMPK is a serine/threonine kinase that forms a complex consisting of $a, \beta$, and $\gamma$ subunits [11]. AMPK is activated when cellular AMP binds to the AMPK- $\gamma$ subunit, leading to phosphorylation of the catalytic AMPK-a subunit. In the case of cellular energy depletion, activated AMPK promotes ATP-producing catabolic pathways (e.g., glycolysis and fatty acid oxidation) and suppresses ATP-consuming anabolic pathways (e.g., gluconeogenesis and lipogenesis), to regulate cellular glucose and lipid metabolism and maintain the energy balance $[12,13]$. In addition to metabolic regulation, AMPK can also control cell growth and autophagy [14]. The molecular mechanisms underlying the cell growth-regulating action of AMPK include inhibition of the mammalian target of rapamycin complex 1 (mTORC 1), which is required for rapid cell proliferation, and activation of tumor suppressor and cell cycle inhibitor proteins [14]. AMPK signaling acts on multiple intracellular pathways, molecules, and transcription factors; therefore, AMPK-activating drugs, such as metformin, may also have beneficial effects in various conditions other than type 2 diabetes and metabolic syndrome.

AMPK and downstream pathways can modulate immune cell function. T cells undergo metabolic reprogramming to adapt to increased energy demands for activation and differentiation; AMPK plays a major role in this process [15]. mTOR, a downstream molecule of AMPK, determines the fate of $T$ cells, i.e., whether they differentiate into effector or regulatory $T$ (Treg) cells [16]. $\mathrm{CD}^{+} \mathrm{T}$ cells require mTORC 1 signaling to differentiate into Th1 and Th17 cells, and mTORC2 signaling to differentiate into Th2 cells, while $\mathrm{CD}^{+} \mathrm{T}$ cells lacking both mTORC1 and mTORC2 signaling differentiate into Treg cells $[16,17]$. The association between the selective expression of mTOR and differentiation of $\mathrm{CD}^{+} \mathrm{T}$ cells is mediated by differential expression of transcription factors, including signal transducer and activator of transcription (STAT). AMPK can directly or indirectly suppress JAK-STAT pathway to exert anti-inflammatory and immunomodulatory effects [18]. AMPK limits the interleukin 6 (IL-6)-mediated inflammatory response by inhibiting STAT3 activation [19]. STAT3 signaling is required not only for Th17 cell differentiation, but also for $T$ follicular helper (Tfh), memory B, and plasma cell differentiation and antigen-specific antibody responses, suggesting that AMPK activators can also target B cell functions [20-23]. Furthermore, AMPK-dependent inhibition of STAT3 and nuclear factor kappa-light-chain-enhancer of activated B cells
(NF-KBs) pathways are involved in the inhibition of monocyte-to-macrophage differentiation, and reduce the production of inflammatory cytokines by macrophages $[24,25]$. Therefore, AMPK activation could be a therapeutic option in autoimmune and inflammatory disorders by modulating multiple intracellular signaling pathways. In this review, we discuss the mechanistic role of metformin in the treatment of rheumatoid arthritis (RA), osteoarthritis (OA), and other autoimmune inflammatory rheumatic diseases.

\section{THERAPEUTIC EFFECTS OF METFORMIN IN RHEUMATOID ARTHRITIS}

RA is a chronic autoimmune disease characterized by synovial hyperplasia and inflammation (synovitis), as well as cartilage and bone destruction in the joints. The interaction of environmental and genetic factors triggers the modification of peptides and failure of immune tolerance, leading to the activation of $T$ and $B$ cells and production of autoantibodies [26]. These activated cells and autoantibodies migrate into the joints and cause an immune response that leads to localized joint inflammation. Innate and adaptive immune cells, cytokines, chemokines, and fibroblast like synoviocytes (FLSs) are involved in the development and perpetuation of synovitis. In addition, FLSs and osteoclasts mediate cartilage and bone destruction, respectively. Therefore, inhibiting these cells in RA patients may have therapeutic benefits.

There is increasing evidence implicating $T$ cells in the pathogenesis of RA. T cells (mostly CD4+ $T$ cells) are abundant in the RA synovium and have intimate contact with other cells [27]. As T cells help B cells to produce autoantibodies, participate in proinflammatory cytokine production, and induce osteoclast activation and bone erosion in RA [28], $T$ cell inhibition might have great benefits in RA treatment. Abatacept (cytotoxic T lymphocyte associated protein 4 [CTLA4]-immunoglobulin), which inhibits the costimulatory signals required for $T$ cell activation, has proven efficacy in RA. However, inhibition of $T$ cells by cyclosporine or anti-CD4 antibodies demonstrated limited efficacy in RA [29]. This suggests that differential targeting of $\mathrm{CD} 4^{+} \mathrm{T}$ cell subtypes, focusing on the deletion of effector T cells, not Treg cells, may be required for the treatment of RA. Th17 cells are a $\mathrm{CD}^{+} \mathrm{T}$ cell subset that play a role in RA pathogenesis by stimulating local inflammation and mediating cartilage and bone destruction [30,31]. However, Treg cells exhibit defec- 
tive function in RA, which is restored by anti-tumor necrosis factor (TNF) treatment [32-34]. These results suggest that an imbalance between Th17 and Treg cells is a hallmark of RA, and maintaining the balance between these cells would reduce disease severity. Modulation of the Th17-Treg cell imbalance is possible by suppressing cytokines, certain cells, or intracellular signaling pathways. Th17-Treg cell imbalance in RA can be reversed by anti-TNF [33-36] and anti-IL-6 receptor $[37,38]$ treatment. STAT3 inhibition using siRNA reduced Th17 cell differentiation and enhanced Treg cell differentiation among $C D 4^{+} T$ cells isolated from the synovial fluids and peripheral blood of RA patients [39]. Therefore, metformin, a STAT3 inhibitor, may exert therapeutic effects in RA by regulating the Th17-Treg cell imbalance. Kang et al. [40] first reported that metformin dose-dependently reduced arthritis severity in mice with collagen-antibody-induced arthritis. Metformin delivered by intraperitoneal injection significantly reduced Th17 cell differentiation, where this effect was associated with the downregulation of MTORC1 and STAT3 through the activation of AMPK. Autoimmune arthritis also improved with oral administration of metformin in mice with collagen-induced arthritis (CIA) [41]. Th17 cell differentiation was decreased and Treg cell differentiation was enhanced by metformin treatment in CIA mice and in vitro experiments.

Synovial pannus is a fibrovascular tissue with a tumor-like, aggressive phenotype that facilitates joint destruction in RA [27]. As a major component of the synovial pannus, FLSs plays a central role in pannus-mediated joint destruction. RA FLSs can proliferate in an anchorage-independent manner, escape contact inhibition, resist apoptosis, and migrate and adhere to the cartilage matrix (and subsequently damage it by producing matrix-degrading enzymes) [42]. Therefore, drugs affecting RA FLSs could have therapeutic efficacy in RA. Metformin has been shown to inhibit the proliferation and migration of RA FLSs $[43,44]$. Metformin-induced cell cycle (G2/M phase) arrest, but not apoptosis, of RA FLSs in vitro [43]. This inhibitory effect on RA FLS proliferation was mediated by phosphoinositide 3-kinase (PI3K)-AKT-mTOR pathway inhibition. Treatment with a PI3K inhibitor also inhibited RA FLS proliferation, whereas the use of a PI3K activator had the opposite effect. These results are in line with the fact that the PI3Kס isoform, expressed by FLSs, is a regulator of FLS growth, migration, and invasion, and may be a potential target for RA therapy $[45,46]$. In addition to the inhibitory effects on the proliferation and migration of
RA FLSs, metformin also downregulated the production of inflammatory cytokines by RA FLSs [44]. Histologically, metformin treatment inhibited synovial hyperplasia, inflammatory cell infiltration, and neovascularization, and downregulated the expression of matrix-degrading enzymes in CIA rat cartilage, confirming its protective effects against synovitis and articular cartilage degradation [47].

Metformin was protective against bone erosion in RA animal models via inhibition of osteoclast differentiation and activity. Osteoclasts are the major cells responsible for bone destruction in RA. Osteoclast differentiation is stimulated by receptor activator of NF-kB ligand (RANKL), which is produced by activated T cells and FLSs $[48,49]$. Local proinflammatory cytokines can also mediate osteoclastogenesis by inducing RANKL or directly stimulating osteoclast precursors [50]. Preclinical studies have found that metformin treatment inhibited osteoclast differentiation and bone-resorbing activity, thereby reducing bone erosions in the joints of CIA models $[41,47,51,52]$. Due to the high energy demands of osteoclast generation and maturation, glycolysis and glutaminolysis are required for osteoclast differentiation and bone resorption [51]. Hypoxia-inducible factor $1 a$ (HIF1a) and c-Myc activation induces glucose and glutamine transporters, and stimulates glycolysis and glutaminolysis, respectively. Furthermore, mTOR is constitutively expressed in osteoclasts, and is required for osteoclastic bone resorption as a nutrient sensor. Treatment with an mTOR inhibitor, Torin1, was found to suppress osteoclast differentiation and bone resorption. Since metformin can suppress mTOR and HIF1a, it could be an inhibitor of osteoclastic bone resorption [41].

Metformin exerts anti-inflammatory effects by regulating autophagy and apoptosis. It was suggested in an earlier study that metformin could exert anti-inflammatory effects by targeting lysosomal proteolysis, similar to chloroquine [53]. The difference was that chloroquine swells lysosomal vesicles and raises the vacuolar $\mathrm{pH}$, whereas metformin binds to metal ions and synergizes their inhibitory action. In another study, metformin improved impaired autophagy to exert anti-inflammatory effects in autoimmune arthritis [54]. Autophagy was observed in a K/BxN serum transfer mouse model, but autophagic flux was markedly impaired. Enhanced autophagic flux by metformin resulted in selective degradation of IKB kinase (a kinase that degrades NF-KB-inhibiting $\mathrm{IKB}$ proteins), thereby suppressing NF-KB signaling and subsequent inflammatory responses. In addition, met- 
formin improved mitochondrial dysfunction in mouse models of RA. A combination of metformin and coenzyme Q10 treatment protected mitochondria against $\mathrm{H}_{2} \mathrm{O}_{2}$-induced apoptosis, by increasing the mitochondrial membrane potential and mitochondrial respiration compared to metformin or coenzyme Q10 alone [55]. Metformin also recovered mitochondrial dysfunction exacerbated by rapamycin treatment [56].

Metformin improved metabolic profiles in in vivo and ex vivo RA models. As metabolic disorders are known to be associated with a poor treatment response in RA $[57,58]$, improving metabolic dysfunction might also ameliorate autoimmune arthritis. Kim et al. [59] found that metformin normalized metabolic dysfunction in obese CIA mice by inducing fibroblast growth factor 21 (FGF21) expression and brown adipose tissue differentiation. Splenocytes co-cultured with brown adipose tissue reduced Th17 cell differentiation, supporting the therapeutic role of metformin in autoimmune arthritis. The relationship between metabolic disorders and RA disease activity was also determined in an Irish RA cohort [60]. Insulin resistance was associated with synovitis in RA patients [60]. The modulation of glucose metabolism in RA FLSs by metformin reduced production of the inflammatory mediators IL-6, IL-8, and monocyte chemoattractant protein 1 (MCP-1) [60], indicating a role of altered glucose metabolism in the suppression of joint inflammation. Moreover, metformin exerted additional anti-inflammatory effects in animal models of RA when concurrently administered with other drugs, such as coenzyme Q10, omega-3, and LMT-28 (an inhibitor of IL-6 signaling) $[55,61,62]$.

Beneficial effects of metformin on RA were demonstrated in clinical studies of RA patients. Using the National Health Insurance Database from Taiwan, patients with RA and type 2 diabetes who received both cyclooxygenase-2 (COX-2) inhibitors and metformin showed lower admission rates during a 10-year follow-up compared to those treated with COX-2 inhibitors alone [63]. Metformin also affected the onset and outcomes of RA. Adherence to metformin treatment was associated with a reduced risk of RA during an 18-year follow-up in a nationwide retrospective cohort study [64]. In a randomized, double-blind, placebo-controlled clinical trial, patients with active RA were treated with additional metformin (1,000 $\mathrm{mg} /$ day) or placebo on background methotrexate therapy [65]. After 12 weeks of treatment, metformin-treated patients showed better clini- cal outcomes than placebo-treated patients, reflected in significant improvement in disease activity and higher rates of remission [65]. These results indicate that metformin could be a beneficial adjunctive therapy in RA. The preclinical and clinical studies regarding the efficacy of metformin in RA are summarized in Table 1.

\section{THERAPEUTIC EFFECTS OF METFORMIN IN OSTEOARTHRITIS}

$\mathrm{OA}$ is a heterogeneous, multifactorial joint disease characterized by cartilage degradation, bone abnormalities (such as osteophyte formation and subchondral bone sclerosis) and low-grade synovial inflammation $[66,67]$. Multiple risk factors, including aging, mechanical stress, trauma, hormonal changes, metabolic disturbances, and genetic predisposition are involved in the pathogenesis of OA. Obesity is an established risk factor for knee, hip, and hand OA [68], and metabolic syndrome and type 2 diabetes are associated with OA $[69,70]$.

Currently, there are no disease-modifying treatments available for OA. As chronic hyperglycemia and insulin resistance induce oxidative stress, chronic low-grade inflammation, and subsequent damage to cartilage matrix [70], metformin might have therapeutic benefits in patients with metabolic OA. In a cohort study using a UK electronic health record database, metformin did not reduce the risk of OA in patients with type 2 diabetes, after adjusting for age and sex [71]. On the other hand, in a case-control study using the Taiwan National Health Insurance Research Database, patients with OA and type 2 diabetes treated with COX2 inhibitors and metformin were at lower risk of joint replacement surgery compared to those treated with COX-2 inhibitors alone [72]. Considering a previous study showing that type 2 diabetes was an independent predictor of severe OA requiring joint replacement [73], metformin treatment might reduce structural damage and lead to better clinical outcomes in OA. This was confirmed in a prospective cohort study performed using Osteoarthritis Initiative (OAI) data, which found that metformin significantly attenuated knee cartilage volume loss (as assessed by magnetic resonance imaging) over a 4-year follow-up in obese OA patients [74]. The mechanisms of action of metformin in OA treatment were further investigated in preclinical studies.

In OA, cartilage degradation is primarily mediated by cat- 
Table 1. Studies regarding metformin efficacy in the treatment of RA

\begin{tabular}{|c|c|}
\hline Study & Results \\
\hline \multicolumn{2}{|l|}{ Preclinical } \\
\hline Lockwood (2010) [53] & Metformin has anti-lysosomal action and increases anti-lysosomal action of $\mathrm{Zn}^{2+}$. \\
\hline Kang et al. (2013) [40] & Metformin attenuated arthritis severity and inhibited Th17 cell differentiation in a CAIA murine model. \\
\hline Indo et al. (2013) [51] & Metformin inhibited osteoclast differentiation in vitro. \\
\hline Son et al. (2014) [41] & $\begin{array}{l}\text { Metformin reduced arthritis severity, suppressed Th17 cell differentiation, enhanced Treg cell differenti- } \\
\text { ation, and suppressed osteoclast differentiation in CIA mice. }\end{array}$ \\
\hline Yan et al. (2015) [54] & $\begin{array}{l}\text { Metformin suppressed arthritis severity and inflammatory cytokine production in a K/BxN serum trans- } \\
\text { fer mouse model by enhancing autophagic flux and suppressing the NF-KB pathway. }\end{array}$ \\
\hline Jhun et al. (2016) [55] & $\begin{array}{l}\text { Combination of metformin and coenzyme Q10 attenuated arthritis severity, suppressed Th17 cell dif- } \\
\text { ferentiation, enhanced Treg cell differentiation, suppressed osteoclast differentiation, and recovered } \\
\text { mitochondrial function in CIA mice to a greater degree compared to treatment with metformin or co- } \\
\text { enzyme Q10 alone. }\end{array}$ \\
\hline Kim et al. (2018) [59] & $\begin{array}{l}\text { Metformin attenuated arthritis severity, decreased Th17 cell differentiation, increased Treg cell differen- } \\
\text { tiation, and improved metabolic profiles in obese CIA mice by inducing FGF21 expression and brown } \\
\text { adipose tissue differentiation. }\end{array}$ \\
\hline Chen et al. (2019) [43] & Metformin inhibited in vitro proliferation of FLS obtained from patients with RA (RA FLS). \\
\hline Chen et al. (2020) [44] & Metformin inhibited in vitro proliferation and migration of RA FLS. \\
\hline Fan et al. (2020) [47] & Metformin improved synovial inflammation and bone and cartilage destruction in joints of CIA rats. \\
\hline Kim et al. (2020) [56] & $\begin{array}{l}\text { Combination of metformin and rapamycin attenuated arthritis severity, regulated Th17 and Treg cell } \\
\text { balance, and improved metabolic profiles in obese CIA mice compared to rapamycin alone or vehicle } \\
\text { treatment. Metformin recovers mitochondrial dysfunction induced by rapamycin. }\end{array}$ \\
\hline Gallagher et al. (2020) [60] & $\begin{array}{l}\text { Alteration of glucose metabolism in synovial fibroblasts by metformin resulted in reduced production of } \\
\text { inflammatory cytokines in ex vivo RA synovial explant cultures and in vitro RA FLS cultures. }\end{array}$ \\
\hline El-Sayyad et al. (2021) [61] & $\begin{array}{l}\text { Combination of metformin and omega-3 attenuated arthritis severity and showed additive anti-inflam- } \\
\text { matory effects in adjuvant-induced arthritis rats compared to metformin or omega-3 alone. }\end{array}$ \\
\hline Park et al. (2021) [62] & $\begin{array}{l}\text { Combination of metformin and LMT-28, an inhibitor of IL- } 6 \text { signaling, ameliorated arthritis severity by } \\
\text { suppressing Th17 cell differentiation, enhancing Treg cell differentiation, and inhibiting IL- } 6 \text { signaling in } \\
\text { CIA mice compared to metformin or LMT- } 28 \text { alone. }\end{array}$ \\
\hline Matsuoka et al. (2021) [52] & Metformin inhibited osteoclastogenesis, inflammatory response, and angiogenesis in vitro. \\
\hline \multicolumn{2}{|l|}{ Clinical } \\
\hline Lu et al. (2019) [63] & $\begin{array}{l}\text { Combination of a COX-2 inhibitor and metformin reduced admission rates of patients with RA and DM } \\
\text { compared to COX-2 inhibitor therapy alone. }\end{array}$ \\
\hline Naffaa et al. (2020) [64] & Adherence to metformin treatment was associated with reduced risk of incident RA in women. \\
\hline Abdallah et al. (2021) [65] & $\begin{array}{l}\text { Patients with RA treated with adjunctive metformin showed a greater rate of achieving ACR20 re- } \\
\text { sponse after } 12 \text { weeks in a randomized, placebo-controlled trial. }\end{array}$ \\
\hline
\end{tabular}

RA, rheumatoid arthritis; CAIA, collagen-antibody induced arthritis; Treg, regulatory T; CIA, collagen-induced arthritis; NF-KB, nuclear factor kappa-light-chain-enhancer of activated B cell; FGF21, fibroblast growth factor 21; FLS, fibroblast like synoviocyte; IL-6, interleukin 6; COX-2, cyclooxygenase-2; DM, diabetes mellitus; ACR20, American College of Rheumatology 20.

abolic factors produced by chondrocytes in response to the proinflammatory cytokines IL-1 $\beta$ and TNF (secreted by synovial lining cells or chondrocytes themselves). Major catabolic factors include matrix metalloproteinases (MMPs) and a disintegrin and metalloproteinase with thrombospondin mo- tifs (ADAMTSs); their expression levels are increased in OA cartilage and synovium [75-78]. Given that both MMPs and ADAMTSs were highly expressed in the lesions of OA cartilage, they might play a major role in cartilage matrix degradation in OA. For instance, MMP-13, the most potent prote- 
ase for type II collagen, was highly expressed in human OA chondrocytes [79], and MMP-13-overexpressing transgenic mice exhibited articular cartilage loss and an OA phenotype [80]. ADAMTS-4 and ADAMTS-5 are the major aggrecanases in human and mouse OA, respectively $[81,82]$, and the deletion of ADAMTS5, and dual deletion of ADAMTS4 and ADAMTS5, prevented cartilage degradation in a surgically induced OA mouse model $[83,84]$. These results indicate that MMP and ADAMTS could be therapeutic targets for the modification of $\mathrm{OA}$.

Metformin reduced the expression of catabolic factors in OA chondrocytes and protected against cartilage degradation in OA animal models. Metformin-treated human OA chondrocytes showed reduced gene expression of the catabolic markers ADAMTS5 and MMP-1 compared to untreated control chondrocytes [85]. The chondroprotective effects of metformin have been demonstrated in an OA mouse model. Articular cartilage degradation developed after surgically induced destabilization of the medial meniscus (DMM) in mice, identified on the basis of higher Osteoarthritis Research Society International (OARSI) scores and smaller cartilage areas [86-88]. Cartilage degradation was significantly reduced in mice administered intragastric or intraarticular metformin [86-88]. Metformin reduced cartilage matrix degradation and stimulated matrix synthesis, as evidenced by a dose-dependent decrease in MMP-13 expression and dose-dependent increase in type II collagen expression in cartilage $[86,87]$. In another study, mouse articular chondrocytes were treated with metformin or vehicle under IL-1 $\beta$ or TNF-a stimulation in vitro, and metformin-treated chondrocytes demonstrated reduced gene expression of MMP-13, MMP-3, ADAMTS4, and ADAMTS5, and increased gene expression of Col2a1 and aggrecan [88]. This inhibition of cartilage catabolism was mediated by AMPK activation. Metformin increased AMPK expression and phosphorylation in murine OA cartilage and chondrocytes [86-88]. The effect of metformin on AMPK activation and MMP-13 inhibition was diminished after an AMPK inhibitor was added to metformin-treated IL-1ß-stimulated mouse chondrocytes [86], and the chondroprotective effect of metformin disappeared in AMPKa1 knockout mice [88]. Given that the deletion of AMPK increased the severity of surgically induced OA in mice [89], these results suggest that maintaining AMPK signaling might be important for preventing OA development and progression, and that metformin could be a potential therapy in OA.
Metformin activated autophagy and inhibited the apoptosis of chondrocytes to attenuate cartilage degradation. There is a direct relation between chondrocyte apoptosis and cartilage degradation [90]. A reduced number of cells and reduced ability to synthesize qualified cartilage matrix contribute to $\mathrm{OA}$ initiation and progression. Chondrocyte apoptosis may be induced by mechanical injury, oxidative stress, impaired mitochondrial function, reduced autophagy, and impaired regulation of cell senescence $[91,92]$. Autophagy inhibition caused OA-like changes in gene expression of chondrocytes, whereas its induction prevented OA-like changes by reducing oxidative stress and apoptosis [93]. Wang et al. [94] demonstrated that metformin reduced oxidative stress and enhanced mitochondrial function in IL-1ß-stimulated mouse chondrocytes by promoting phosphatase and tensin homolog (PTEN)-induced putative kinase protein 1 (PINK1)/Parkin-dependent mitophagy. Metformin enhanced PINK1/Parkin-dependent mitophagy, an autophagic elimination of damaged mitochondria, via upregulation of sirtuin 3 (SIRT3), a mitochondrial deacetylase. Activation of SIRT3-mediated PINK1/Parkin-dependent mitophagy had protective effects against OA cartilage catabolism. Another action of metformin was activation of AMPK/ SIRT1-mediated autophagy in chondrocytes [95]. Metformin attenuated cartilage degradation, and also enhanced autophagy and inhibited chondrocyte apoptosis, in the articular cartilage tissue of DMM-induced OA mice. Silencing AMPKa2, but not AMPKa1, reduced the expression of SIRT1 and abolished metformin-induced autophagy activation, suggesting that the AMPKa2-SIRT1 pathway was involved in the autophagy-augmenting effects of metformin in chondrocytes. The regulation of autophagy and apoptosis by metformin was replicated in monosodium iodoacetate-induced OA mice and in vitro human OA chondrocytes [96]. Metformin also delayed aging of articular chondrocytes, as demonstrated by reduced expression of cell senescence markers [87].

Metformin decreased synovitis and osteophyte formation in OA animal models. Histologic grading and micro-computed tomography analysis demonstrated synovial hyperplasia and osteophyte formation at 6 and 12 weeks after DMM surgery in mice [88]. Metformin markedly reduced the synovitis score, and osteophyte size and maturity [88]. IL-1 $\beta$ and TNF produced by OA chondrocytes stimulate chondrocytes and synovial cells to produce other proinflammatory mediators and catabolic factors, leading to cartilage de- 
struction. Cartilage degradation products in turn amplify synovial inflammation, and the activated synovium produces proteases that damage adjacent cartilage, forming a vicious cycle [97]. Therefore, decreasing synovitis might prevent or delay OA progression. Metformin reduced joint and systemic inflammation by inhibiting NF-KB, which was involved in inflammatory OA cascades $[88,98,99]$. In addition to reducing synovitis and structural damage, metformin also decreased pain levels in OA animal models $[86,88,96]$. The inhibitory action of metformin on pain-related markers in dorsal root ganglion and articular chondrocytes was also mediated by AMPK signaling activation [88]. Moreover, concomitant administration of metformin augmented the anti-inflammatory and chondroprotective effects of other treatments, such as mesenchymal stem cells (MSCs) and COX-2 inhibitors, in experimental OA, suggesting clinical applications for OA treatment $[96,100]$. The preclinical and clinical studies assessing the efficacy of metformin in OA are summarized in Table 2.

\section{THERAPEUTIC EFFECTS OF METFORMIN IN OTHER AUTOIMMUNE INFLAMMATORY RHEUMATIC DISEASES}

Systemic lupus erythematosus (SLE) is an autoimmune disease that affects multiple organs and is characterized by activation of the type 1 interferon (IFN) pathway, autoreactive $T$ and $B$ cell proliferation, autoantibody production, and immune complex deposition $[101,102]$. CD4 ${ }^{+} T$ cells from mouse models of and patients with SLE exhibited increased glycolysis and mitochondrial oxidative phosphorylation, reflecting CD4 ${ }^{+} T$ cell activation [103]. Combined treatment with 2-deoxy-D-glucose, a glycolysis inhibitor, and metformin, a mitochondrial oxidative phosphorylation inhibitor, normalized lupus $\mathrm{CD}^{+}{ }^{+} \mathrm{T}$ cell effector functions in vitro and reversed the lupus phenotype in mice. A subsequent study found that pyruvate oxidation and oxidative phosphorylation, rather than the pyruvate reduction to lactate, were important for $\mathrm{CD}^{+} \mathrm{T}$ cell activation in lupus-prone mice [104]. The inhibition of pyruvate reduction to lactate, which is an anaerobic process, did not reverse or prevent the lupus phenotype in mice. These results indicate that targeting glucose oxidation can be a novel therapeutic strategy for SLE.

Metformin can inhibit the type 1 IFN pathway. Wang et al. [105] observed enhanced release of mitochondrial DNA
(mtDNA) from neutrophil extracellular traps (NETs) and increased levels of antibodies against mtDNA in SLE patients. Correlated with SLE disease activity and lupus nephritis, mtDNA and anti-mtDNA induced IFN-a from plasmacytoid dendritic cells (pDCs) to a significantly greater degree compared to any other DNA antigen or autoantibody. Metformin treatment downregulated NET mtDNA release from neutrophils and decreased IFN-a production from pDCs [105]. Metformin also inhibited the type 1 IFN response in $\mathrm{CD}^{+} \mathrm{T}$ cells in healthy individuals and SLE patients, by suppressing STAT1 phosphorylation and its downstream signaling [106]. These effects of metformin relied on an inhibitory action against mitochondrial oxidative phosphorylation and were independent of AMPK and mTORC1 signaling.

Metformin controlled adaptive immune responses in SLE animal models. In experiments with murine SLE, metformin decreased autoantibody titers and target organ inflammation, including lupus nephritis [107]. Metformin inhibited germinal center reaction, controlled B cell differentiation into plasma cells, and regulated $T$ cell differentiation by reducing Tfh and Th17 cells and increasing Treg cells. The regulation of $B$ and $T$ cells by metformin was associated with AMPK activation, and mTOR and STAT3 inhibition. In addition, metformin enhanced the immunomodulatory effects of other treatments. A recent study by Jang et al. [108] demonstrated enhanced immunoregulatory actions of metformin-treated MSCs compared to metformin-untreated MSCs. Metformin upregulated STAT1 expression to enhance the immunoregulatory actions of MSCs; these effects were mediated by the AMPK and mTOR pathways. The administration of metformin-treated MSCs improved dysregulated $B$ and $T$ cell responses and the lupus phenotype in mice [108]. Another study investigated the therapeutic effect of metformin in combination with abatacept in murine SLE [109]. Although abatacept did not have any efficacy in lupus nephritis, the combination of metformin and abatacept, administered early in the disease course, reduced renal pathology and prevented lupus nephritis, as well as suppressing $\mathrm{CD}^{+} \mathrm{T}$ cell effector subsets. When metformin (500 mg three times per day) was added to standard treatment with glucocorticoids and/or immunosuppressive agents in SLE patients in randomized clinical trials, the risk of SLE flares and required dose of glucocorticoids were reduced $[105,110]$.

Therapeutic effects of metformin were also investigated in Sjögren's syndrome [111], scleroderma [112-115], anky- 
Table 2. Studies regarding metformin efficacy in the treatment of OA

\begin{tabular}{|c|c|}
\hline Study & Results \\
\hline \multicolumn{2}{|l|}{ Preclinical } \\
\hline Wang et al. (2019) [94] & $\begin{array}{l}\text { Metformin inhibited IL-1ß-induced oxidative stress and mitochondrial dysfunction in mouse chondrocytes } \\
\text { via the activation of SIRT3-mediated PINK1/Parkin-dependent mitophagy. }\end{array}$ \\
\hline Park et al. (2019) [100] & $\begin{array}{l}\text { Metformin-treated Ad-hMSCs inhibited inflammatory and catabolic process of IL-1B-stimulated OA chon- } \\
\text { drocytes and revealed antinociceptive and chondroprotective effects in MIA-induced OA rats compared } \\
\text { to metformin-untreated Ad-hMSCs. }\end{array}$ \\
\hline Schadler et al. (2020) [85] & $\begin{array}{l}\text { Metformin-treated human OA chondrocytes had reduced gene expressions of catabolic markers, such as } \\
\text { ADAMTS5 and MMP1, compared to untreated control samples. }\end{array}$ \\
\hline Li et al. (2020) [86] & $\begin{array}{l}\text { Metformin attenuated cartilage degradation and modulated pain behavior in a DMM-induced OA mouse } \\
\text { model as well as reduced MMP-13 expression and increased type II collagen expression in IL-1ß-stimu- } \\
\text { lated mouse chondrocytes. }\end{array}$ \\
\hline Feng et al. (2020) [87] & $\begin{array}{l}\text { Metformin reduced cartilage degradation and aging in a DMM-induced OA mouse model and IL-1ß-stim- } \\
\text { ulated mouse cartilage explants. }\end{array}$ \\
\hline Li et al. (2020) [88] & $\begin{array}{l}\text { Metformin reduced cartilage degradation, synovitis, and osteophyte formation and attenuated pain in } \\
\text { a DMM-induced OA mouse model and inhibited catabolic markers and enhanced anabolic markers in } \\
\text { TNF-a or IL-1 } \beta \text {-stimulated mouse chondrocytes. These effects were abolished in AMPKa1 KO mice. }\end{array}$ \\
\hline Wang et al. (2020) [95] & $\begin{array}{l}\text { Metformin attenuated cartilage degradation, activated autophagy, and inhibited apoptosis in a DMM-in- } \\
\text { duced OA mouse model and IL-1 } \beta \text { - stimulated mouse chondrocytes via the AMPKa2-SIRT1 pathway. }\end{array}$ \\
\hline Dawood et al. (2020) [98] & $\begin{array}{l}\text { Induction of diabetes caused articular cartilage loss in rats, which was inhibited by metformin treatment. } \\
\text { Metformin inhibited hyperglycemia and markers associated with inflammation and oxidative stress. }\end{array}$ \\
\hline Zhang et al. (2020) [99] & $\begin{array}{l}\text { Metformin suppressed ECM catabolism, inflammation, and apoptosis in IL-1ß-stimulated mouse chondro- } \\
\text { cytes. }\end{array}$ \\
\hline Na et al. (2021) [96] & $\begin{array}{l}\text { Metformin reduced pain and cartilage degradation in MIA-induced OA rats and inhibited apoptosis and } \\
\text { activated autophagy in IL-1ß-stimulated human OA chondrocytes. }\end{array}$ \\
\hline \multicolumn{2}{|l|}{ Clinical } \\
\hline Barnett et al. (2017) [71] & Exposure to metformin treatment had no association with OA risk in patients with type 2 diabetes. \\
\hline Lu et al. (2018) [72] & $\begin{array}{l}\text { Combination of a COX-2 inhibitor and metformin reduced rate of joint replacement in patients with OA } \\
\text { and DM compared to COX-2 inhibitor therapy alone. }\end{array}$ \\
\hline Wang et al. (2019) [74] & Metformin use was associated with less knee cartilage volume loss over 4 years in obese people with OA. \\
\hline
\end{tabular}

OA, osteoarthritis; IL-1 $\beta$, interleukin-1 $\beta$; SIRT, sirtuin; PINK1, phosphatase and tensin homolog (PTEN)-induced putative kinase protein 1; Ad-hMSC, adipose tissue-derived human mesenchymal stem cell; MIA, modosodium iodoacetate; ADAMTS5, a disintegrin and metalloproteinase with thrombospondin motifs 5; MMP, matrix metalloproteinase; DMM, destabilization of medial meniscus; TNF-a; tumor necrosis factor-a; AMPK, AMP-activated kinase; KO, knockout; ECM, extracellular matrix; COX-2, cyclooxygenase-2; DM, diabetes mellitus.

losing spondylitis [116], and gout [117]. Metformin reversed salivary gland inflammation and hypofunction in murine Sjögren's syndrome by controlling imbalanced T and B cell differentiation [111]. To examine the effects of metformin on scleroderma, a mouse model of bleomycin-induced skin fibrosis was employed [112-115]. Metformin attenuated skin fibrosis in mice, as evidenced by reductions in skin thickness, collagen deposition, and numbers of myofibroblasts. Metformin-treated mice had increased Treg cells, and decreased Th17 cells, Tfh cells, germinal center B cells, and proinflammatory cytokine production, along with mTOR and STAT3 suppression in skin fibroblasts. As gout is an inflammatory arthritis induced by monosodium urate (MSU) crystals deposited in tissues, including joints, and where the inflammation is mediated by mTOR signaling in monocytes, inhibition of mTOR might have therapeutic benefits in gout [117]. mTOR inhibition by metformin resulted in reduced MSU crystal-induced monocyte death (pyroptosis) and in- 
flammation in vitro; gout patients exhibited a decreased frequency of gout attacks [117].

\section{CONCLUSIONS}

The antidiabetic mechanisms of metformin are still being elucidated, despite its widespread, long-term use in type 2 diabetes. As metformin regulates cell metabolism, proliferation, growth, and autophagy, it might have disease-modifying effects in various other conditions. Metformin has shown therapeutic benefits in obesity, aging, cardiovascular diseases, liver diseases, renal diseases, and cancers [118]. In this review, we focused on the therapeutic applications of metformin in autoimmunity and inflammation, and dis- cussed how metformin impacts RA, OA, and other autoimmune inflammatory rheumatic diseases. Potential molecular and cellular mechanisms of action of metformin in RA and $\mathrm{OA}$ are summarized in Fig. 1. Metformin inhibits the proliferation and migration of FLS, regulates Th17 and Treg cell differentiation, suppresses osteoclast differentiation and activity, and reduces inflammatory cytokine production. Metformin can also enhance autophagy and improve mitochondrial functions. Metformin decreases chondrocyte apoptosis and cartilage catabolism. These effects are mediated by the modulation of multiple interacting intracellular pathways, such as PI3K-AKT-mTOR, mTOR, STAT3, HIF1a, NF-KB, and SIRT signaling. By controlling synovitis and joint destruction in $\mathrm{RA}$, and attenuating cartilage degradation in $\mathrm{OA}$ in vitro and in vivo, metformin has demonstrated therapeutic
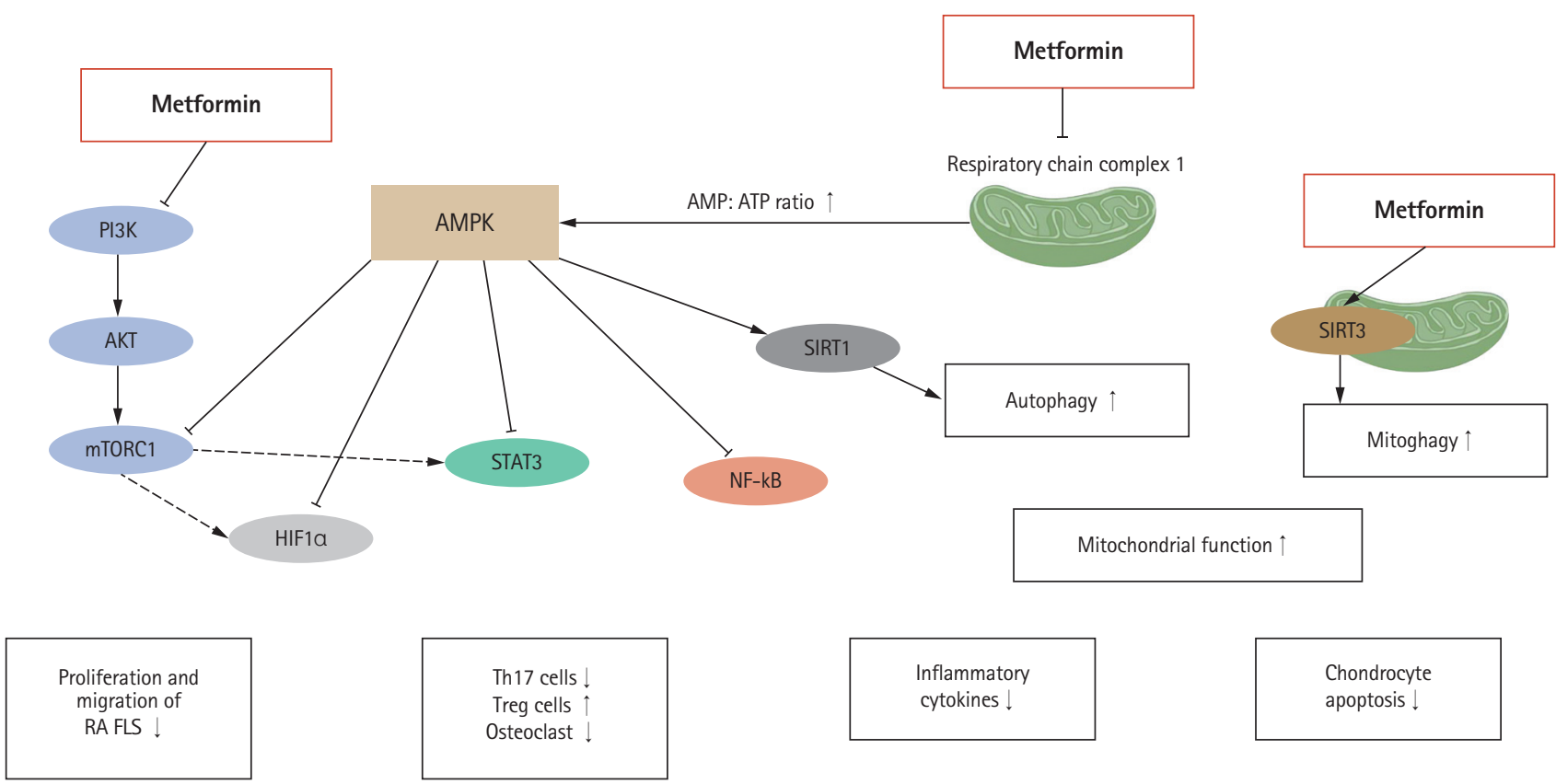

Chondrocyte apoptosis $\downarrow$

\section{Cartilage catabolic factors} (MMP, ADAMTS) $\downarrow$

Figure 1. Potential molecular and cellular mechanisms of metformin in rheumatoid arthritis (RA) and osteoarthritis (OA). Metformin inhibits mitochondrial respiratory chain complex 1, which results in depleted energy status, as represented by the increased adenosine monophosphate (AMP)/adenosine triphosphate (ATP) ratio. This causes the activation of AMP-activated protein kinase (AMPK). AMPK modulates cellular metabolism, proliferation, growth, and autophagy through multiple intracellular signaling pathways. Metformin inhibits proliferation and migration of RA fibroblast like synoviocytes (FLSs), regulates Th17 and regulatory T (Treg) cell differentiation, suppresses osteoclast differentiation, and reduces inflammatory cytokine production, thus controlling synovitis and joint destruction in RA. Metformin also enhances autophagy, mitophagy, and mitochondrial function, leading to decreased apoptosis. Inhibitory actions of metformin against chondrocyte apoptosis and cartilage catabolism attenuate cartilage degradation in OA. PI3K, phosphoinositide 3-kinase; mTORC, mammalian target of rapamycin complex; HIF1a, hypoxia-inducible factor 1a; STAT, signal transducer and activator of transcription; NFKB, nuclear factor kappa-light-chain-enhancer of activated B cell; SIRT, sirtuin; MMP, matrix metalloproteinase; ADAMTS, a disintegrin and metalloproteinase with thrombospondin motif. 
benefits in both diseases. Metformin enhances the immunomodulatory, anti-inflammatory, and chondroprotective effects of conventional treatments, suggesting its role as an adjunctive therapy. In clinical studies, metformin treatment was associated with a reduced risk and severity of RA and OA. Metformin reduced the rates of admission and joint replacement surgery, and attenuated cartilage damage in obese and diabetic patients with RA or OA. These results suggest that metformin may have clinical efficacy in RA and OA; however, further clinical studies in large populations are warranted to support its use in a clinical setting.

\section{Conflict of interest}

No potential conflict of interest relevant to this article was reported.

\section{Acknowledgments}

This work was supported by a grant of the Korea Health Technology R\&D Project through the Korea Health Industry Development Institute (KHIDI), funded by the Ministry of Health and Welfare, Republic of Korea (grant number HI20C1496), and was also supported by the National Research Foundation of Korea (NRF) grant funded by the Korea government (Ministry of Science and ICT) (No. 2019R1G1A1100421).

\section{REFERENCES}

1. Foretz M, Guigas B, Viollet B. Understanding the glucoregulatory mechanisms of metformin in type 2 diabetes mellitus. Nat Rev Endocrinol 2019;15:569-589.

2. Zhou G, Myers R, Li Y, et al. Role of AMP-activated protein kinase in mechanism of metformin action. J Clin Invest 2001;108:1167-1174.

3. Shaw RJ, Lamia KA, Vasquez D, et al. The kinase LKB1 mediates glucose homeostasis in liver and therapeutic effects of metformin. Science 2005;310:1642-1646.

4. El-Mir MY, Nogueira V, Fontaine E, Averet N, Rigoulet M, Leverve $X$. Dimethylbiguanide inhibits cell respiration via an indirect effect targeted on the respiratory chain complex I. J Biol Chem 2000;275:223-228.

5. Owen MR, Doran E, Halestrap AP. Evidence that metformin exerts its anti-diabetic effects through inhibition of complex 1 of the mitochondrial respiratory chain. Biochem J 2000;348(Pt 3):607-614.
6. Stephenne $X$, Foretz $M$, Taleux N, et al. Metformin activates AMP-activated protein kinase in primary human hepatocytes by decreasing cellular energy status. Diabetologia 2011;54:3101-3110.

7. Foretz M, Hebrard S, Leclerc J, et al. Metformin inhibits hepatic gluconeogenesis in mice independently of the LKB1/AMPK pathway via a decrease in hepatic energy state. J Clin Invest 2010;120:2355-2369.

8. Hunter RW, Hughey CC, Lantier L, et al. Metformin reduces liver glucose production by inhibition of fructose-1-6-bisphosphatase. Nat Med 2018;24:1395-1406.

9. Miller RA, Chu Q, Xie J, Foretz M, Viollet B, Birnbaum MJ. Biguanides suppress hepatic glucagon signalling by decreasing production of cyclic AMP. Nature 2013;494:256-260.

10. Madiraju AK, Erion DM, Rahimi Y, et al. Metformin suppresses gluconeogenesis by inhibiting mitochondrial glycerophosphate dehydrogenase. Nature 2014;510:542-546.

11. Kim J, Yang G, Kim Y, Kim J, Ha J. AMPK activators: mechanisms of action and physiological activities. Exp Mol Med 2016;48:e224.

12. Long YC, Zierath JR. AMP-activated protein kinase signaling in metabolic regulation. J Clin Invest 2006;116:1776-1783.

13. Towler MC, Hardie DG. AMP-activated protein kinase in metabolic control and insulin signaling. Circ Res 2007;100:328341.

14. Mihaylova MM, Shaw RJ. The AMPK signalling pathway coordinates cell growth, autophagy and metabolism. Nat Cell Biol 2011;13:1016-1023.

15. Blagih J, Coulombe F, Vincent EE, et al. The energy sensor AMPK regulates $T$ cell metabolic adaptation and effector responses in vivo. Immunity 2015;42:41-54.

16. Delgoffe GM, Kole TP, Zheng Y, et al. The mTOR kinase differentially regulates effector and regulatory $\mathrm{T}$ cell lineage commitment. Immunity 2009;30:832-844.

17. Delgoffe GM, Pollizzi KN, Waickman AT, et al. The kinase mTOR regulates the differentiation of helper $T$ cells through the selective activation of signaling by mTORC 1 and $\mathrm{mTORC} 2$. Nat Immunol 2011;12:295-303.

18. Speirs C, Williams J, Riches K, Salt IP, Palmer TM. Linking energy sensing to suppression of JAK-STAT signalling: a potential route for repurposing AMPK activators? Pharmacol Res 2018;128:88-100.

19. Nerstedt A, Johansson A, Andersson CX, Cansby E, Smith U, Mahlapuu M. AMP-activated protein kinase inhibits IL-6-stimulated inflammatory response in human liver cells by suppressing phosphorylation of signal transducer and activator of 
transcription 3 (STAT3). Diabetologia 2010;53:2406-2416.

20. Ma CS, Avery DT, Chan A, et al. Functional STAT3 deficiency compromises the generation of human $T$ follicular helper cells. Blood 2012;119:3997-4008.

21. Ray JP, Marshall HD, Laidlaw BJ, Staron MM, Kaech SM, Craft J. Transcription factor STAT3 and type I interferons are corepressive insulators for differentiation of follicular helper and $T$ helper 1 cells. Immunity 2014;40:367-377.

22. Avery DT, Deenick EK, Ma CS, et al. B cell-intrinsic signaling through IL-21 receptor and STAT3 is required for establishing long-lived antibody responses in humans. J Exp Med 2010;207:155-171.

23. Deenick EK, Avery DT, Chan A, et al. Naive and memory human $B$ cells have distinct requirements for STAT3 activation to differentiate into antibody-secreting plasma cells. J Exp Med 2013;210:2739-2753.

24. Vasamsetti SB, Karnewar S, Kanugula AK, Thatipalli AR, Kumar JM, Kotamraju S. Metformin inhibits monocyte-to-macrophage differentiation via AMPK-mediated inhibition of STAT3 activation: potential role in atherosclerosis. Diabetes 2015;64:2028-2041.

25. Kim J, Kwak HJ, Cha JY, et al. Metformin suppresses lipopolysaccharide (LPS)-induced inflammatory response in murine macrophages via activating transcription factor-3 (ATF-3) induction. J Biol Chem 2014;289:23246-23255.

26. Mclnnes IB, Schett $G$. Pathogenetic insights from the treatment of rheumatoid arthritis. Lancet 2017;389:2328-2337.

27. Bartok B, Firestein GS. Fibroblast-like synoviocytes: key effector cells in rheumatoid arthritis. Immunol Rev 2010;233:233255.

28. Firestein GS, Zvaifler NJ. How important are T cells in chronic rheumatoid synovitis?: II. T cell-independent mechanisms from beginning to end. Arthritis Rheum 2002;46:298-308.

29. Panayi GS. Even though T-cell-directed trials have been of limited success, is there reason for optimism? Nat Clin Pract Rheumatol 2006;2:58-59.

30. Moran EM, Mullan R, McCormick J, et al. Human rheumatoid arthritis tissue production of IL-17A drives matrix and cartilage degradation: synergy with tumour necrosis factor-alpha, oncostatin $\mathrm{M}$ and response to biologic therapies. Arthritis Res Ther 2009;11:R113.

31. Sato K, Suematsu A, Okamoto K, et al. Th17 functions as an osteoclastogenic helper $T$ cell subset that links $T$ cell activation and bone destruction. J Exp Med 2006;203:2673-2682.

32. Valencia X, Stephens G, Goldbach-Mansky R, Wilson M, Shevach EM, Lipsky PE. TNF downmodulates the function of human $C D 4^{+}$CD25hi T-regulatory cells. Blood 2006;108:253261.

33. Ehrenstein MR, Evans JG, Singh A, et al. Compromised function of regulatory $T$ cells in rheumatoid arthritis and reversal by anti-TNFalpha therapy. J Exp Med 2004;200:277-285.

34. Nie H, Zheng Y, Li R, et al. Phosphorylation of FOXP3 controls regulatory $T$ cell function and is inhibited by TNF-a in rheumatoid arthritis. Nat Med 2013;19:322-328.

35. Aravena $O$, Pesce B, Soto $L$, et al. Anti-TNF therapy in patients with rheumatoid arthritis decreases Th1 and Th17 cell populations and expands IFN- - -producing NK cell and regulatory $T$ cell subsets. Immunobiology 2011;216:1256-1263.

36. McGovern JL, Nguyen DX, Notley CA, Mauri C, Isenberg DA, Ehrenstein MR. Th17 cells are restrained by Treg cells via the inhibition of interleukin- 6 in patients with rheumatoid arthritis responding to anti-tumor necrosis factor antibody therapy. Arthritis Rheum 2012;64:3129-3138.

37. Samson M, Audia S, Janikashvili N, et al. Brief report: inhibition of interleukin-6 function corrects Th17/Treg cell imbalance in patients with rheumatoid arthritis. Arthritis Rheum 2012;64:2499-2503.

38. Pesce B, Soto L, Sabugo F, et al. Effect of interleukin-6 receptor blockade on the balance between regulatory $T$ cells and $T$ helper type 17 cells in rheumatoid arthritis patients. Clin Exp Immunol 2013;171:237-242.

39. Ju JH, Heo YJ, Cho ML, et al. Modulation of STAT-3 in rheumatoid synovial T cells suppresses Th17 differentiation and increases the proportion of Treg cells. Arthritis Rheum 2012;64:3543-3552.

40. Kang KY, Kim YK, Yi H, et al. Metformin downregulates Th17 cells differentiation and attenuates murine autoimmune arthritis. Int Immunopharmacol 2013;16:85-92.

41. Son HJ, Lee J, Lee SY, et al. Metformin attenuates experimental autoimmune arthritis through reciprocal regulation of Th17/Treg balance and osteoclastogenesis. Mediators Inflamm 2014;2014:973986.

42. You S, Koh JH, Leng L, Kim WU, Bucala R. The tumor-like phenotype of rheumatoid synovium: molecular profiling and prospects for precision medicine. Arthritis Rheumatol 2018;70:637-652.

43. Chen K, Lin ZW, He SM, et al. Metformin inhibits the proliferation of rheumatoid arthritis fibroblast-like synoviocytes through IGF-IR/PI3KJAKT/m-TOR pathway. Biomed Pharmacother 2019;115:108875.

44. Chen Y, Qiu F, Yu B, et al. Metformin, an AMPK activator, inhibits activation of FLSs but promotes HAPLN1 secretion. Mol 
Ther Methods Clin Dev 2020;17:1202-1214.

45. Bartok B, Boyle DL, Liu Y, et al. PI3 kinase $\delta$ is a key regulator of synoviocyte function in rheumatoid arthritis. Am J Pathol 2012;180:1906-1916.

46. Bartok B, Hammaker D, Firestein GS. Phosphoinositide 3-kinase $\delta$ regulates migration and invasion of synoviocytes in rheumatoid arthritis. J Immunol 2014;192:2063-2070.

47. Fan KJ, Wu J, Wang QS, Xu BX, Zhao FT, Wang TY. Metformin inhibits inflammation and bone destruction in collagen-induced arthritis in rats. Ann Transl Med 2020;8:1565.

48. Jung SM, Kim KW, Yang CW, Park SH, Ju JH. Cytokine-mediated bone destruction in rheumatoid arthritis. J Immunol Res 2014;2014:263625.

49. Kim HR, Kim KW, Kim BM, Lee KA, Lee SH. N-acetyl-I-cysteine controls osteoclastogenesis through regulating Th17 differentiation and RANKL production in rheumatoid arthritis. Korean J Intern Med 2019;34:210-219.

50. Schett G, Gravallese E. Bone erosion in rheumatoid arthritis: mechanisms, diagnosis and treatment. Nat Rev Rheumatol 2012;8:656-664.

51. Indo $Y$, Takeshita S, Ishii KA, et al. Metabolic regulation of osteoclast differentiation and function. J Bone Miner Res 2013;28:2392-2399.

52. Matsuoka Y, Morimoto S, Fujishiro M, et al. Metformin repositioning in rheumatoid arthritis. Clin Exp Rheumatol 2021;39:763-768.

53. Lockwood TD. The lysosome among targets of metformin: new anti-inflammatory uses for an old drug? Expert Opin Ther Targets 2010;14:467-478.

54. Yan H, Zhou HF, Hu Y, Pham CT. Suppression of experimental arthritis through AMP-activated protein kinase activation and autophagy modulation. J Rheum Dis Treat 2015;1:5.

55. Jhun J, Lee S, Kim SY, et al. Combination therapy with metformin and coenzyme Q10 in murine experimental autoimmune arthritis. Immunopharmacol Immunotoxicol 2016;38:103-112.

56. Kim EK, Min HK, Lee SY, et al. Metformin rescues rapamycin-induced mitochondrial dysfunction and attenuates rheumatoid arthritis with metabolic syndrome. Arthritis Res Ther 2020;22:77.

57. Koh JH, Park YJ, Lee S, et al. Distinct urinary metabolic profile in rheumatoid arthritis patients: a possible link between diet and arthritis phenotype. J Rheum Dis 2019;26:46-56.

58. Son KM, Kang SH, Seo Yl, Kim HA. Association of body composition with disease activity and disability in rheumatoid arthritis. Korean J Intern Med 2021;36:214-222.
59. Kim EK, Lee SH, Lee SY, et al. Metformin ameliorates experimental-obesity-associated autoimmune arthritis by inducing FGF21 expression and brown adipocyte differentiation. Exp Mol Med 2018;50:e432.

60. Gallagher L, Cregan S, Biniecka M, et al. Insulin-resistant pathways are associated with disease activity in rheumatoid arthritis and are subject to disease modification through metabolic reprogramming: a potential novel therapeutic approach. Arthritis Rheumatol 2020;72:896-902.

61. El-Sayyad SM, Ali MA, Kandil LS, Ragab GM, Abdelhamid Ibrahim SS. Metformin and omega-3 fish oil elicit anti-inflammatory effects via modulation of some dysregulated micro RNAs expression and signaling pathways in experimental induced arthritis. Int Immunopharmacol 2021;92:107362.

62. Park YH, Jang YJ, Choi Y, et al. Combination of LMT-28 and metformin improves beneficial anti-inflammatory effect in collagen-induced arthritis. Pharmacology 2021;106:53-59.

63. Lu CH, Chung $\mathrm{CH}$, Lee $\mathrm{CH}$, et al. Combination of COX-2 inhibitor and metformin attenuates rate of admission in patients with rheumatoid arthritis and diabetes in Taiwan. Medicine (Baltimore) 2019;98:e17371.

64. Naffaa ME, Rosenberg V, Watad A, et al. Adherence to metformin and the onset of rheumatoid arthritis: a population-based cohort study. Scand J Rheumatol 2020;49:173180.

65. Abdallah MS, Alarfaj SJ, Saif DS, et al. The AMPK modulator metformin as adjunct to methotrexate in patients with rheumatoid arthritis: a proof-of-concept, randomized, double-blind, placebo-controlled trial. Int Immunopharmacol 2021;95:107575.

66. Bijlsma JW, Berenbaum F, Lafeber FP. Osteoarthritis: an update with relevance for clinical practice. Lancet 2011;377:21152126.

67. Deveza LA, Loeser RF. Is osteoarthritis one disease or a collection of many? Rheumatology (Oxford) 2018;57(suppl 4):iv34iv42.

68. Reyes C, Leyland KM, Peat G, Cooper C, Arden NK, Prieto-Alhambra $D$. Association between overweight and obesity and risk of clinically diagnosed knee, hip, and hand osteoarthritis: a population-based cohort study. Arthritis Rheumatol 2016;68:1869-1875.

69. Zhuo Q, Yang W, Chen J, Wang Y. Metabolic syndrome meets osteoarthritis. Nat Rev Rheumatol 2012;8:729-737.

70. Veronese N, Cooper C, Reginster JY, et al. Type 2 diabetes mellitus and osteoarthritis. Semin Arthritis Rheum 2019;49:919. 
71. Barnett LA, Jordan KP, Edwards JJ, van der Windt DA. Does metformin protect against osteoarthritis? An electronic health record cohort study. Prim Health Care Res Dev 2017;18:623628.

72. Lu CH, Chung $\mathrm{CH}$, Lee $\mathrm{CH}$, et al. Combination COX-2 inhibitor and metformin attenuate rate of joint replacement in osteoarthritis with diabetes: a nationwide, retrospective, matched-cohort study in Taiwan. PLoS One 2018;13:e0191242.

73. Schett $G$, Kleyer A, Perricone C, et al. Diabetes is an independent predictor for severe osteoarthritis: results from a longitudinal cohort study. Diabetes Care 2013;36:403-409.

74. Wang Y, Hussain SM, Wluka AE, et al. Association between metformin use and disease progression in obese people with knee osteoarthritis: data from the Osteoarthritis Initiative. A prospective cohort study. Arthritis Res Ther 2019;21:127.

75. Kevorkian L, Young DA, Darrah C, et al. Expression profiling of metalloproteinases and their inhibitors in cartilage. Arthritis Rheum 2004;50:131-141.

76. Davidson RK, Waters JG, Kevorkian L, et al. Expression profiling of metalloproteinases and their inhibitors in synovium and cartilage. Arthritis Res Ther 2006;8:R124.

77. Abramson SB. Osteoarthritis and nitric oxide. Osteoarthritis Cartilage 2008;16 Suppl 2:S15-S20.

78. Attur M, Al-Mussawir HE, Patel J, et al. Prostaglandin E2 exerts catabolic effects in osteoarthritis cartilage: evidence for signaling via the EP4 receptor. J Immunol 2008;181:50825088.

79. Roach HI, Yamada N, Cheung KS, et al. Association between the abnormal expression of matrix-degrading enzymes by human osteoarthritic chondrocytes and demethylation of specific $C p G$ sites in the promoter regions. Arthritis Rheum 2005;52:3110-3124.

80. Neuhold LA, Killar L, Zhao W, et al. Postnatal expression in hyaline cartilage of constitutively active human collagenase-3 (MMP-13) induces osteoarthritis in mice. J Clin Invest 2001;107:35-44.

81. Stanton H, Rogerson FM, East CJ, et al. ADAMTS5 is the major aggrecanase in mouse cartilage in vivo and in vitro. Nature 2005;434:648-652.

82. Yatabe T, Mochizuki S, Takizawa M, et al. Hyaluronan inhibits expression of ADAMTS4 (aggrecanase-1) in human osteoarthritic chondrocytes. Ann Rheum Dis 2009;68:1051-1058.

83. Glasson SS, Askew R, Sheppard B, et al. Deletion of active ADAMTS5 prevents cartilage degradation in a murine model of osteoarthritis. Nature 2005;434:644-648.

84. Majumdar MK, Askew R, Schelling S, et al. Double-knockout of ADAMTS- 4 and ADAMTS- 5 in mice results in physiologically normal animals and prevents the progression of osteoarthritis. Arthritis Rheum 2007;56:3670-3674.

85. Schadler P, Lohberger B, Stundl N, et al. The effect of body mass index and metformin on matrix gene expression in arthritic primary human chondrocytes. Cartilage 2021;13(2 Suppl):1004S-1018S.

86. Li $H$, Ding $X$, Terkeltaub R, et al. Exploration of metformin as novel therapy for osteoarthritis: preventing cartilage degeneration and reducing pain behavior. Arthritis Res Ther 2020;22:34.

87. Feng $X$, Pan J, Li J, et al. Metformin attenuates cartilage degeneration in an experimental osteoarthritis model by regulating AMPK/mTOR. Aging (Albany NY) 2020;12:1087-1103.

88. Li J, Zhang B, Liu WX, et al. Metformin limits osteoarthritis development and progression through activation of AMPK signalling. Ann Rheum Dis 2020;79:635-645.

89. Zhou S, Lu W, Chen L, et al. AMPK deficiency in chondrocytes accelerated the progression of instability-induced and ageing-associated osteoarthritis in adult mice. Sci Rep 2017;7:43245.

90. Thomas CM, Fuller CJ, Whittles CE, Sharif M. Chondrocyte death by apoptosis is associated with cartilage matrix degradation. Osteoarthritis Cartilage 2007;15:27-34.

91. Carames B, Taniguchi N, Otsuki S, Blanco FJ, Lotz M. Autophagy is a protective mechanism in normal cartilage, and its aging-related loss is linked with cell death and osteoarthritis. Arthritis Rheum 2010;62:791-801.

92. Loeser RF, Collins JA, Diekman BO. Ageing and the pathogenesis of osteoarthritis. Nat Rev Rheumatol 2016;12:412-420.

93. Sasaki H, Takayama K, Matsushita T, et al. Autophagy modulates osteoarthritis-related gene expression in human chondrocytes. Arthritis Rheum 2012;64:1920-1928.

94. Wang C, Yang Y, Zhang Y, Liu J, Yao Z, Zhang C. Protective effects of metformin against osteoarthritis through upregulation of SIRT3-mediated PINK1/Parkin-dependent mitophagy in primary chondrocytes. Biosci Trends 2019;12:605-612.

95. Wang C, Yao Z, Zhang Y, et al. Metformin mitigates cartilage degradation by activating AMPK/SIRT1-mediated autophagy in a mouse osteoarthritis model. Front Pharmacol 2020;11:1114.

96. Na HS, Kwon JY, Lee SY, et al. Metformin attenuates monosodium-iodoacetate-induced osteoarthritis via regulation of pain mediators and the autophagy-lysosomal pathway. Cells 2021;10:681.

97. Sellam J, Berenbaum F. The role of synovitis in pathophysiolo- 
gy and clinical symptoms of osteoarthritis. Nat Rev Rheumatol 2010;6:625-635.

98. Dawood AF, Alzamil N, Ebrahim HA, et al. Metformin pretreatment suppresses alterations to the articular cartilage ultrastructure and knee joint tissue damage secondary to type 2 diabetes mellitus in rats. Ultrastruct Pathol 2020;44:273-282.

99. Zhang M, Liu Y, Huan Z, Wang Y, Xu J. Metformin protects chondrocytes against IL-1 $\beta$ induced injury by regulation of the AMPK/NF-kB signaling pathway. Pharmazie 2020;75:632-636.

100. Park MJ, Moon SJ, Baek JA, et al. Metformin augments anti-inflammatory and chondroprotective properties of mesenchymal stem cells in experimental osteoarthritis. J Immunol 2019;203:127-136.

101. Tsokos GC, Lo MS, Costa Reis P, Sullivan KE. New insights into the immunopathogenesis of systemic lupus erythematosus. Nat Rev Rheumatol 2016;12:716-730.

102. Lee YH, Song GG. Associations between circulating interleukin-17 levels and systemic lupus erythematosus and between interleukin-17 gene polymorphisms and disease susceptibility: a meta-analysis. J Rheum Dis 2020;27:37-44.

103. Yin $Y$, Choi SC, Xu Z, et al. Normalization of CD4 ${ }^{+} \mathrm{T}$ cell metabolism reverses lupus. Sci Transl Med 2015;7:274ra18.

104. Yin Y, Choi SC, Xu Z, et al. Glucose oxidation is critical for $\mathrm{CD}^{+} \mathrm{T}$ cell activation in a mouse model of systemic lupus erythematosus. J Immunol 2016;196:80-90.

105. Wang H, Li T, Chen S, Gu Y, Ye S. Neutrophil extracellular trap mitochondrial DNA and its autoantibody in systemic lupus erythematosus and a proof-of-concept trial of metformin. Arthritis Rheumatol 2015;67:3190-3200.

106. Titov AA, Baker HV, Brusko TM, Sobel ES, Morel L. Metformin inhibits the type 1 IFN response in human $\mathrm{CD}^{+} \mathrm{T}$ cells. J Immunol 2019;203:338-348.

107. Lee SY, Moon SJ, Kim EK, et al. Metformin suppresses systemic autoimmunity in Roquinsan/san mice through inhibiting $B$ cell differentiation into plasma cells via regulation of AMPK/ mTOR/STAT3. J Immunol 2017;198:2661-2670.

108. Jang SG, Lee J, Hong SM, Kwok SK, Cho ML, Park SH. Metformin enhances the immunomodulatory potential of adi- pose-derived mesenchymal stem cells through STAT1 in an animal model of lupus. Rheumatology (Oxford) 2020;59:14261438.

109. Cornaby C, Elshikha AS, Teng X, et al. Efficacy of the combination of metformin and CTLA4Ig in the (NZB $\times$ NZW) F1 mouse model of lupus nephritis. Immunohorizons 2020;4:319-331.

110. Sun F, Geng S, Wang H, et al. Effects of metformin on disease flares in patients with systemic lupus erythematosus: post hoc analyses from two randomised trials. Lupus Sci Med 2020;7:e000429.

111. Kim JW, Kim SM, Park JS, et al. Metformin improves salivary gland inflammation and hypofunction in murine Sjögren's syndrome. Arthritis Res Ther 2019;21:136.

112. Karatas A, Celik C, Oz B, et al. Secukinumab and metformin ameliorate dermal fibrosis by decreasing tissue interleukin-17 levels in bleomycin-induced dermal fibrosis. Int J Rheum Dis 2021;24:795-802.

113. Moon J, Lee SY, Choi JW, et al. Metformin ameliorates scleroderma via inhibiting Th17 cells and reducing mTOR-STAT3 signaling in skin fibroblasts. J Transl Med 2021;19:192.

114. Ursini F, Grembiale RD, D'Antona L, et al. Oral metformin ameliorates bleomycin-induced skin fibrosis. J Invest Dermatol 2016;136:1892-1894.

115. Wang Y, Zhang S, Liang Z, et al. Metformin attenuates bleomycin-induced scleroderma by regulating the balance of Treg/ Teff cells and reducing spleen germinal center formation. Mol Immunol 2019;114:72-80.

116. Qin X, Jiang T, Liu S, et al. Effect of metformin on ossification and inflammation of fibroblasts in ankylosing spondylitis: an in vitro study. J Cell Biochem 2018;119:1074-1082.

117. Vazirpanah N, Ottria A, van der Linden M, et al. mTOR inhibition by metformin impacts monosodium urate crystal-induced inflammation and cell death in gout: a prelude to a new addon therapy? Ann Rheum Dis 2019;78:663-671.

118. Lv Z, Guo Y. Metformin and its benefits for various diseases. Front Endocrinol (Lausanne) 2020;11:191. 